2020, Instituto Mexicano de Tecnología del Agua

DOI: $10.24850 / j-$ tyca-2020-03-01

Artículos

\title{
Estimación de áreas vulnerables a inundaciones en zonas urbanas: Morelia, Michoacán, México
}

\section{Estimating areas vulnerable to flooding in urban zones: Morelia, Michoacán, Mexico}

Anastasia Alarcón Neva ${ }^{1}$

Jesús Chávez Morales²

Óscar Luis Palacios Vélez ${ }^{3}$

Laura Alicia Ibáñez Castillo4, ORCID: https://orcid.org/0000-0001-9287$655 X$

${ }^{1}$ Colegio de Postgraduados, Montecillo, Texcoco, Estado de México, México, alarcon.anastasia@colpox.mx

${ }^{2}$ Colegio de Postgraduados, Montecillo, Texcoco, Estado de México, México, chavezje@colpos.mx

${ }^{3}$ Colegio de Postgraduados, Montecillo, Texcoco, Estado de México, México, opalacio@colpos.mx

4Universidad Autónoma Chapingo, Postgrado en Ingeniería Agrícola y Uso Integral del Agua, carretera México-Texcoco, Chapingo, Texcoco, Estado de México, México, libacas@gmail.com 
Autora para correspondencia: Anastasia Alarcón Neva, alarcon.anastasia@colpox.mx

\section{Resumen}

Se determinaron áreas vulnerables a inundaciones en la ciudad de Morelia Michoacán, causadas por el río Grande de Morelia que la cruza; esto, mediante la aplicación de un modelo hidrológico en su cuenca hidrológica y otro hidráulico sobre el río, en su tramo por la ciudad.

La modelación hidrológica se realizó con HEC-HMS, considerando las características de las subcuencas como área, tiempo de retraso y valores de curvas de escurrimientos. Las precipitaciones se incorporaron mediante hietogramas para septiembre de 2013. Se calibró el modelo, tomando como base un hidrograma aforado a la salida de la cuenca y adecuando valores de curva número de escurrimientos hasta tener un ajuste aceptable entre el hidrograma calculado por el modelo y el aforado a la salida de la cuenca; el ajuste se evaluó mediante los criterios de Nash-Sutcliffe, RMSE y el coeficiente de determinación.

Con el modelo hidrológico calibrado, se identificó un evento con duración de 24 horas y se sustituyeron las precipitaciones por hietogramas asociados con los periodos de retorno de 100 y 500 años, que se estimaron mediante la función de distribución de probabilidad Gumbel; se modelaron ambos eventos y se obtuvieron hidrogramas.

La modelación hidráulica del río Grande se realizó con IBER, retomando los hidrogramas para cada periodo de retorno, topografía del cauce y coeficientes de rugosidad de Manning. Los resultados de esta

Tecnología y ciencias del agua, ISSN 2007-2422, 11(3), 01-26. DOI: $10.24850 / j$-tyca-2020-0301 
última modelación permitieron identificar las zonas vulnerables a inundaciones producidas por avenidas asociadas con los periodos de retorno considerados.

Palabras clave: modelo, hietograma, estación meteorológica automática, hidrograma, Gumbel, periodo de retorno.

\section{Abstract}

We determined areas of Morelia, Michoacán, that are vulnerable to flooding caused by the river Río Grande de Morelia, which crosses the city. A hydrological model in the river's basin and another hydraulic model on the stretch of river that goes through the city were applied.

The hydrological model was carried out with HEC-HMS considering the characteristics of the sub-basins such as area, lag time and number of runoff curves. The precipitations were incorporated with hyetographs for September 2013. The model was calibrated based on a gauged hydrograph at the outlet of the basin and adjusting the values of the runoff curve number until an acceptable fit was obtained between the hydrograph calculated by the model and that gauged at the basin's exit. The fit was evaluated with the criteria Nash-Sutcliffe, RMSE and Coefficient of Determination.

With the calibrated hydrological model, an event lasting 24 hours was identified and the precipitations for hyetographs were substituted by those associated with 100- and 500-year return periods estimated with the Gumbel Probability Distribution. Both events were modeled, and hydrographs were obtained. 
Hydraulic modeling of the section of the Río Grande River that crosses the city was carried out with IBER, using the hydrographs for each return period, channel topography and Manning roughness coefficients. The results of this last modeling allowed identification of the areas vulnerable to flooding produced by rises associated with the return periods considered.

Keywords: Model, hyetograph, automatic weather station, hydrograph, Gumbel PDF, return periods, recurrence interval.

Recibido: 05/07/2018

Aceptado: 23/08/2019

\section{Introducción}

Una inundación es un desbordamiento del agua por arriba de los niveles normales de una corriente u otro cuerpo de agua, o la acumulación de agua sobre áreas que normalmente no están sumergidas (WMO, 2011); por lo general, las lluvias extraordinarias son la principal causante de inundaciones que ponen en peligro vida y bienes materiales (Kidd \& Huffman, 2011). 
Para la modelación del proceso lluvia-escurrimiento se utilizan, entre otras técnicas, los modelos hidrológicos, como el Hydrologic Engineering Center-Hydrologic Modeling System (HEC-HMS) (HEC-HMS, 2017) (USACE, 2016); por otra parte, para el tránsito de avenidas en cauces y llanuras de inundación se emplean modelos de tipo hidráulico (Fuentes-Mariles \& Franco, 1999). Un modelo de simulación hidráulica bidimensional en cauces es IBER, desarrollado por el Instituto en Dinámica Fluvial e Ingeniería Hidrológica (FLUMEN, 2010), que tiene la bondad de considerar la topografía de cauces y llanuras de inundación en una malla.

En el caso de la ciudad de Morelia, el crecimiento poblacional ha provocado que zonas con riesgo a inundaciones sean ocupadas por la mancha urbana (Corona-Morales, 2009). En esta urbe se han presentado múltiples inundaciones, ocasionando pérdidas económicas y de infraestructura (Conagua, 2016). En este trabajo se determinaron zonas vulnerables a inundaciones para Morelia; se propone el uso de modelos matemáticos.

\section{Metodología}




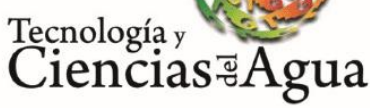

2020, Instituto Mexicano de Tecnología del Agua

Open Access bajo la licencia CC BY-NC-SA 4.0

(https://creativecommons.org/licenses/by-nc-sa/4.0/)

Se delimitó la cuenca hasta la Estación Hidrométrica Convencional El Plan, utilizando HEC-GeoHMS (HEC-GeoHMS, 2017). Dicho programa generó un esquema conformado por subcuencas, ríos y uniones; asimismo, se determinaron parámetros, como el tiempo de retraso y valor del número de curva de escurrimiento a nivel subcuenca, principalmente.

El análisis de información meteorológica se realizó con la disponible de las estaciones meteorológicas convencionales (EMC), que son un conjunto de instrumentos colocados a la intemperie que permiten medir las variaciones del clima, y estaciones meteorológicas automáticas (EMA), que son las conformadas por un grupo de sensores que registran y transmiten información meteorológica de forma automática.

Con el registro de las precipitaciones para el mes de septiembre de 2013 de las EMA se generó un hietograma adimensional y que fue base para generar hietogramas en las EMC. Por otra parte, con el registro histórico de las EMC se analizaron las máximas en 24 horas, con lo cual se estimaron precipitaciones para los periodos de retorno ( $T r)$ de 100 y 500 años, con la función de distribución de probabilidad Gumbel.

El análisis de información hidrométrica consistió en la revisión del registro histórico de aforos de la estación hidrométrica convencional (EHC) El Plan, con el que se generó un hidrograma.

La modelación hidrológica se realizó con HEC-HMS (2017), donde se utilizó el esquema de la cuenca. A HEC-HMS se ingresaron valores de áreas, número de curva de escurrimientos y tiempo de retraso. La simulación hidrológica se llevó a cabo en dos partes: la primera, para calibrar el modelo utilizando como base el hidrograma observado en la 
EHC El Plan, y la segunda para la modelación de eventos máximos en 24 horas para los periodos de retorno de 100 y 500 años.

Se realizó la modelación hidráulica con IBER, con una malla irregular con información de geometría de cauces, coeficiente de Manning e hidrogramas para cada cauce tributario del río Grande. Con los resultados del modelo hidráulico se identificaron las zonas vulnerables a inundaciones.

\section{Resultados}

La cuenca hidrológica hasta la EHC El Plan, clave 12588 (19 49' 10" N y $\left.101^{\circ} 00^{\prime} 40^{\prime \prime} \mathrm{O}\right)$, se localiza en la porción noroeste del estado de Michoacán y abarca un área de $1578.36 \mathrm{~km}^{2}$. A su vez, la cuenca se dividió en 14 subcuencas, tal como se presentan en la Figura 1. 
2020, Instituto Mexicano de Tecnología del Agua

Tecnología y

Open Access bajola licencia CC BY-NC-SA 4.0

(https://creativecommons.org/licenses/by-nc-sa/4.0/)

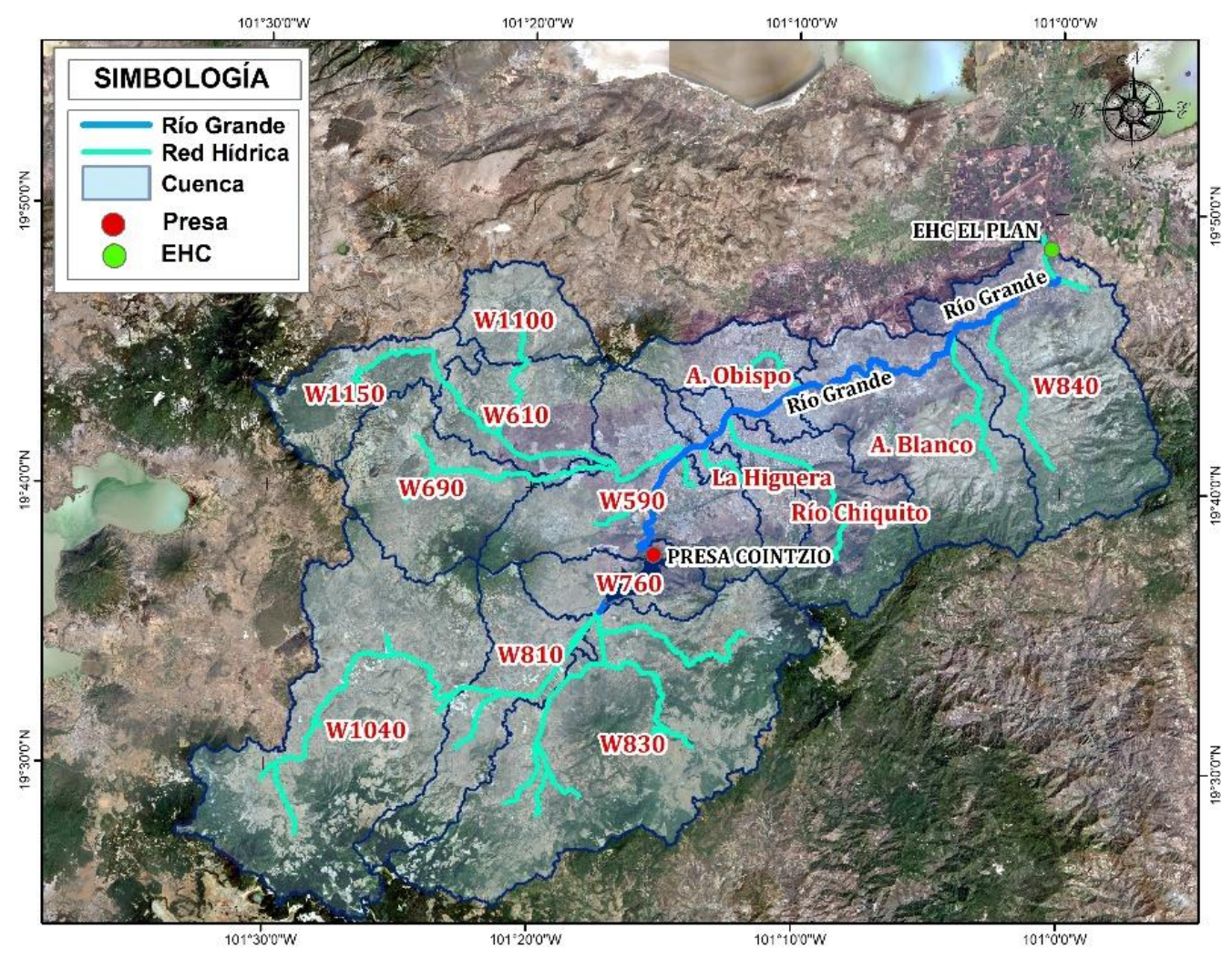

Figura 1. Localización de las subcuencas.

La hidrografía en Morelia está conformada, principalmente, por el río Grande, al que se le integran afluentes, como el río Itzícuaro, río Chiquito, arroyo La Higuera y arroyo El Obispo. En la zona alta del río Grande se localiza la presa Cointzio ( $19^{\circ} 37^{\prime} 48^{\prime \prime} \mathrm{N}$ y $\left.101^{\circ} 15^{\prime} 27^{\prime \prime} \mathrm{O}\right)$.

Los tiempos de concentración se calcularon con la fórmula de Kirpich (Conagua, 2011) y los tiempos de retraso se calcularon considerando el $60 \%$ del tiempo de concentración (USACE, 2016).

En cuanto a los valores de número de curva de escurrimiento, se obtuvieron realizando la discretización de la distribución edafológica de 
Tecnología y

Ciencias $\approx$ Agua
2020, Instituto Mexicano de Tecnología del Agua

Open Access bajo la licencia CC BY-NC-SA 4.0

(https://creativecommons.org/licenses/by-nc-sa/4.0/)

las cartas vectoriales con las que se determinó textura, unidad y subunidad del suelo predominante; con esto se determinó el grupo hidrológico de suelo $(A, B, C$ o D). Después, con las cartas de uso de suelo y vegetación se determinaron los valores de número de curva de escurrimiento en función de la condición de cobertura vegetal de la superficie. En la Tabla 1 se concentran las principales características por subcuenca.

Tabla 1. Características por subcuenca.

\begin{tabular}{|c|c|c|c|c|}
\hline Subcuenca & $\begin{array}{c}\text { Área } \\
\left(\mathbf{k m}^{2}\right)\end{array}$ & $\begin{array}{c}\text { Tiempo de } \\
\text { concentración } \\
\text { (min) }\end{array}$ & $\begin{array}{l}\text { Tiempo de } \\
\text { retraso }(\mathrm{min})\end{array}$ & $\begin{array}{c}\text { Número de } \\
\text { curva de } \\
\text { escurrimiento } \\
\text { (adimensional) }\end{array}$ \\
\hline W1040 & 260.48 & 308.51 & 185.1 & 77.55 \\
\hline W810 & 88.00 & 276.26 & 165.8 & 77.74 \\
\hline W830 & 283.27 & 274.47 & 164.7 & 79.08 \\
\hline W760 & 45.97 & 143.31 & 120.3 & 79.94 \\
\hline W1150 & 60.11 & 36.68 & 95.0 & 78.06 \\
\hline W1100 & 45.47 & 83.18 & 86.0 & 81.18 \\
\hline W610 & 68.79 & 158.25 & 213.1 & 81.27 \\
\hline W690 & 99.49 & 200.49 & 22.0 & 79.05 \\
\hline W590 & 126.77 & 355.13 & 49.9 & 83.94 \\
\hline W840 & 32.29 & 215.64 & 43.6 & 79.9 \\
\hline La Higuera & 95.19 & 72.64 & 123.5 & 91.27 \\
\hline Río Chiquito & 149.39 & 205.77 & 282.6 & 82.7 \\
\hline
\end{tabular}

Tecnología y ciencias del agua, ISSN 2007-2422,11(3), 01-26. DOI: 10.24850/j-tyca-2020-0301 
Tecnología y

\section{Ciencias $\stackrel{\Xi}{\approx}$ gua}

2020, Instituto Mexicano de Tecnología del Agua

Open Access bajola licencia CC BY-NC-SA 4.0

(https://creativecommons.org/licenses/by-nc-sa/4.0/)

\begin{tabular}{|l|c|c|c|c|}
\hline $\begin{array}{l}\text { Arroyo } \\
\text { Blanco }\end{array}$ & 54.19 & 174.35 & 104.6 & 83.23 \\
\hline $\begin{array}{l}\text { Arroyo } \\
\text { Obispo }\end{array}$ & 168.94 & 471.07 & 129.4 & 90.19 \\
\hline
\end{tabular}

El análisis meteorológico consistió en identificar las estaciones cercanas a la cuenca, resultando seis EMC y dos EMA; para conocer la influencia de éstas por subcuenca se generaron isoyetas para septiembre de 2013 (Figura 2).

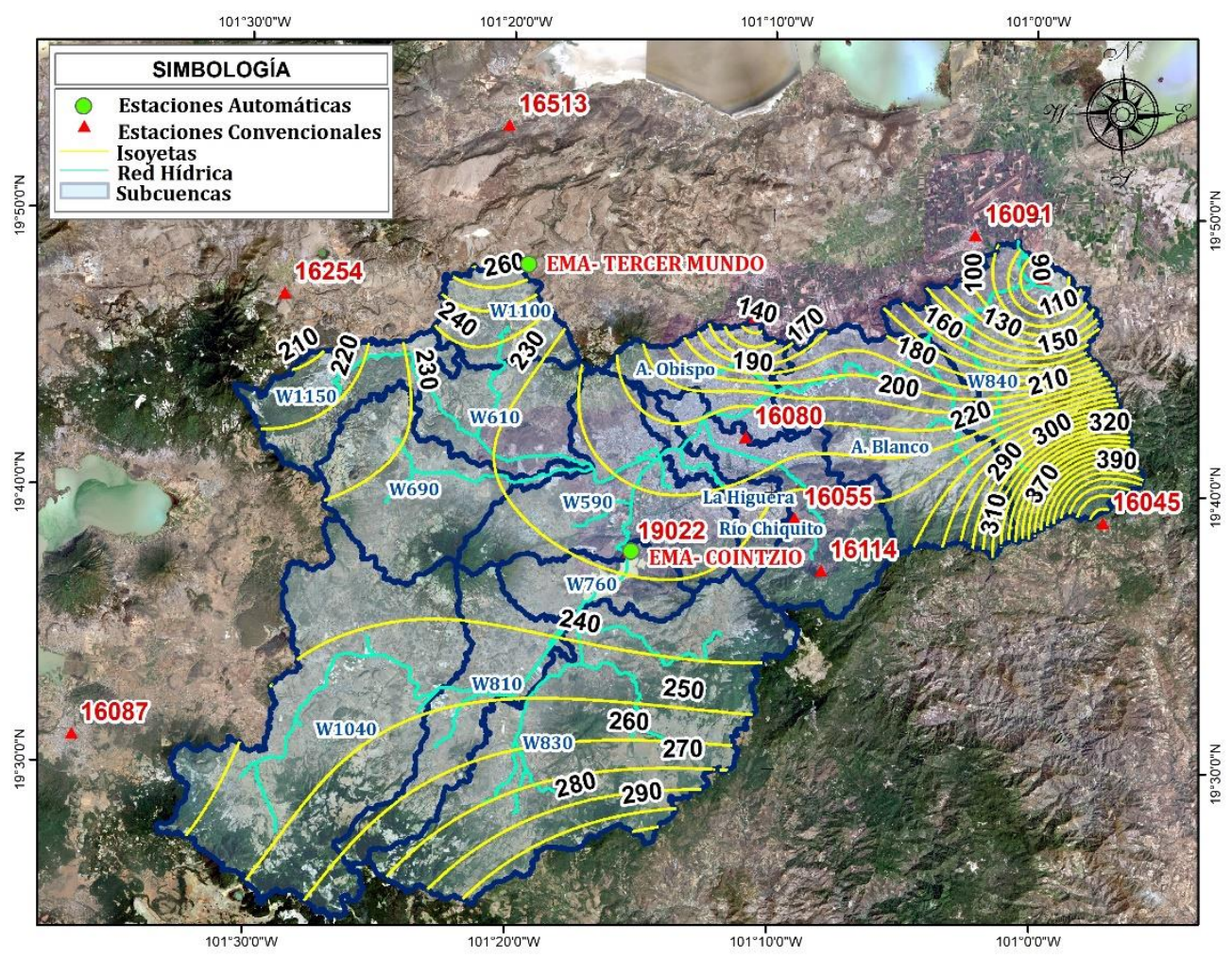

Figura 2. Isoyetas para el mes de septiembre de 2013. 
Tecnología y

Ciencias $₫$ Agua
2020, Instituto Mexicano de Tecnología del Agua

Open Access bajo la licencia CC BY-NC-SA 4.0

(https://creativecommons.org/licenses/by-nc-sa/4.0/)

Del análisis de isoyetas, se asignó una estación de influencia a cada subcuenca, quedando la relación que se presenta en la Tabla 2.

Tabla 2. Estaciones seleccionadas por subcuenca.

\begin{tabular}{|c|c|c|c|}
\hline Subcuenca & Estación & Subcuenca & Estación \\
\hline W1040 & $\begin{array}{l}\text { EMA-Tercer } \\
\text { Mundo/16513 El Jacal }\end{array}$ & W690 & $\begin{array}{l}\text { EMA-Tercer } \\
\text { Mundo/16513 El Jacal }\end{array}$ \\
\hline W810 & $\begin{array}{l}\text { EMA-Tercer } \\
\text { Mundo/16513 El Jacal }\end{array}$ & W590 & 16105-Quirio \\
\hline W830 & $\begin{array}{l}\text { EMA-Cointzio/16022 } \\
\text { Cointzio }\end{array}$ & W840 & 16105-Quirio \\
\hline W760 & $\begin{array}{l}\text { EMA-Cointzio/16022 } \\
\text { Cointzio }\end{array}$ & La Higuera & $\begin{array}{l}\text { EMA-Cointzio/16022 } \\
\text { Cointzio }\end{array}$ \\
\hline W1100 & $\begin{array}{l}\text { EMA-Tercer } \\
\text { Mundo/16513 El Jacal }\end{array}$ & Río Chiquito & 16080-Morelia (OBS) \\
\hline W1150 & 16254-Teremendo & Arroyo Blanco & 16512- El Colegio \\
\hline W610 & $\begin{array}{l}\text { EMA-Cointzio/16022 } \\
\text { Cointzio }\end{array}$ & El Obispo & 16512-El Colegio \\
\hline
\end{tabular}

No se tuvo acceso a los datos pluviométricos para generar hietogramas con intervalos menores a 24 horas. Sin embargo, se retomó la metodología de López et al. (2012), partiendo del registro de precipitación en algunas EMA vecinas fuera o dentro de la cuenca (que por lo general reportan precipitación cada 10 minutos), se asume que la distribución en 24 horas de los valores diarios en las EMC es similar al de las EMA. 
Con el reporte de las dos EMA para septiembre de 2013 cada 10 minutos, se retomó la distribución temporal de la precipitación y se generó un hietograma mensual en cada EMA (dividiendo las ordenadas del hietograma observado en las EMA, entre la precipitación total registrada en el mismo mes). Estos hietogramas se multiplicaron por la lámina de precipitación total reportada en las EMC para el mismo mes, obteniendo así hietogramas en las EMC.

Con el registro histórico en las EMC para el periodo de 1986 a 2014, de precipitaciones máximas en 24 horas y la función Gumbel (Aparicio, 1992), se obtuvieron las precipitaciones máximas esperadas para los periodos de retornos de 100 y 500 años para las EMC (Tabla 3).

Tabla 3. Precipitaciones máximas $(\mathrm{mm})$ en 24 horas para periodos de retorno de 100 y 500 años.

\begin{tabular}{|c|c|c|c|c|c|c|}
\hline $\begin{array}{c}\text { Tr } \\
\text { (Años) }\end{array}$ & $\begin{array}{c}\mathbf{1 6 5 1 3} \\
\text { El Jacal }\end{array}$ & $\begin{array}{c}\mathbf{1 6 5 1 2} \\
\text { El Colegio }\end{array}$ & $\begin{array}{c}\mathbf{1 6 2 5 4} \\
\text { Teremendo }\end{array}$ & $\begin{array}{c}\mathbf{1 6 0 8 0} \\
\text { Morelia (OBS) }\end{array}$ & $\begin{array}{c}\mathbf{1 6 0 2 2} \\
\text { Cointzio }\end{array}$ & $\begin{array}{c}\mathbf{1 6 1 0 5} \\
\text { Quirio }\end{array}$ \\
\hline 100 & 94.00 & 86.16 & 83.06 & 101.01 & 85.04 & 73.23 \\
\hline 500 & 114.47 & 102.60 & 99.78 & 121.55 & 102.11 & 86.78 \\
\hline
\end{tabular}

En el análisis hidrométrico se identificó que la única EHC con información disponible y útil fue la 12588 El Plan; con su reporte de aforos de septiembre de 2013 se generó un hidrograma (Figura 3). 
2020, Instituto Mexicano de Tecnología del Agua

Tecnología y

Open Access bajola licencia CCBY-NC-SA 4.0

(https://creativecommons.org/licenses/by-nc-sa/4.0/)

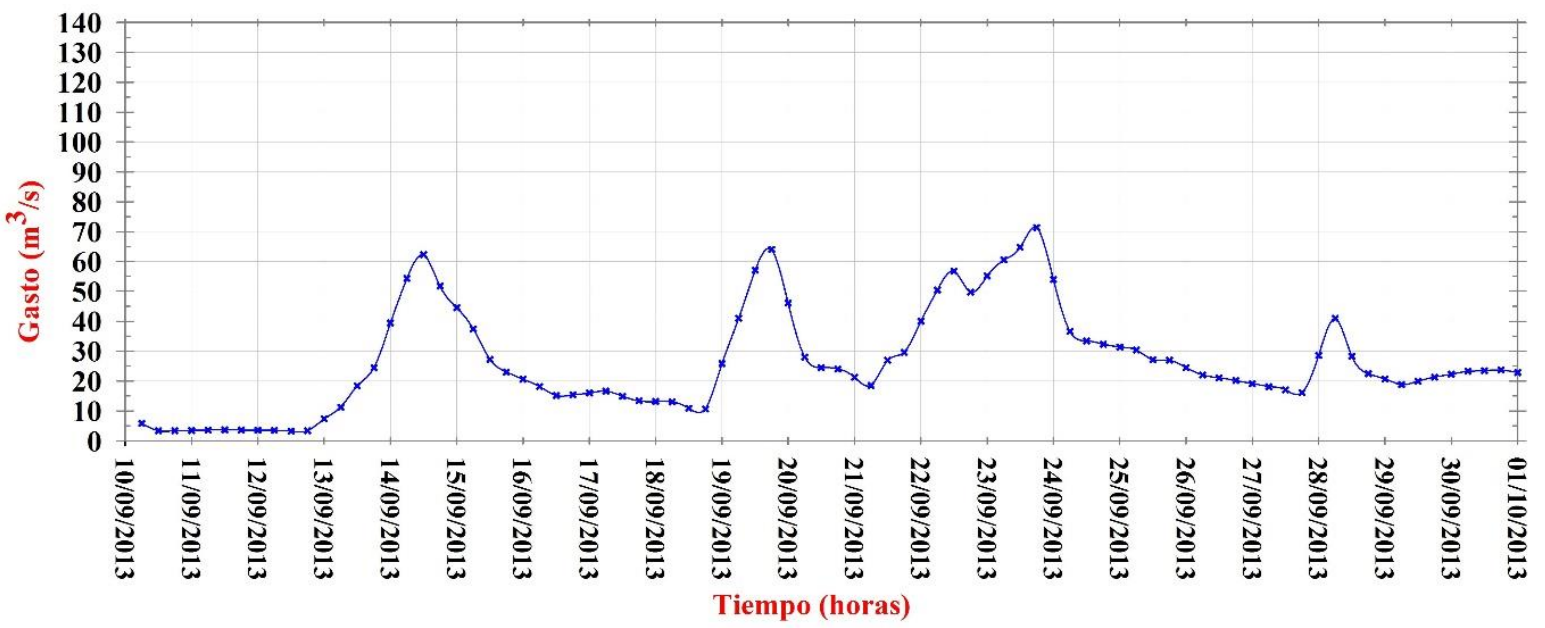

Figura 3. Hidrograma para el mes de septiembre del año 2013 en la EHC El Plan.

\section{Modelación hidrológica}

La modelación hidrológica se realizó en HEC-HMS (USACE, 2016), este programa retomó el esquema de la cuenca, integrado por las subcuencas, ríos, uniones y presa Cointzio. Los parámetros que se ingresaron al modelo por subcuenca fueron los valores de área, número de curva de escurrimientos y tiempo de retraso. También se integró la presa Cointzio como obra de regulación de avenidas máximas, considerándola a presa Ilena. 
Tecnología y

Ciencias $\stackrel{\varpi}{\triangleleft}$ gua
2020, Instituto Mexicano de Tecnología del Agua

Open Access bajo la licencia CC BY-NC-SA 4.0

(https://creativecommons.org/licenses/by-nc-sa/4.0/)

En el módulo de meteorología se ingresaron los hietogramas del mes de septiembre; se corrió el modelo para todo el mes, con intervalos de 10 minutos y se obtuvo un hidrograma simulado, que al compararlo con el hidrograma observado (aforado) en la EHC El Plan resultó que los picos de avenidas coinciden; sin embargo, el modelo los sobreestimó. Para calibrar el modelo, se ajustaron los valores de número de curvas de escurrimientos para cada subcuenca hasta llegar a un valor óptimo, cuidando que estos valores no sobrepasaran el $15 \%$, respecto al valor inicial propuesto (Tabla 4).

Tabla 4. Valores de número de curvas de escurrimiento ajustados a nivel subcuenca (adimensional).

\begin{tabular}{|c|c|c|c|}
\hline \multirow{2}{*}{ Subcuenca } & \multicolumn{2}{|c|}{ Número de curva de escurrimiento } & \multirow{2}{*}{$\begin{array}{c}\text { Diferencia } \\
\text { (\%) }\end{array}$} \\
\cline { 2 - 3 } & Estimada & Ajustada & 7.76 \\
\hline W1040 & 77.55 & 71.53 & 11.90 \\
\hline W810 & 77.74 & 68.51 & 6.00 \\
\hline W830 & 79.08 & 74.42 & 13.98 \\
\hline W760 & 79.94 & 69.09 & 13.65 \\
\hline W1150 & 78.06 & 67.47 & 6.16 \\
\hline W1100 & 81.18 & 76.40 & 12.83 \\
\hline W610 & 81.27 & 71.32 & 5.99 \\
\hline W690 & 79.05 & 74.40 & 6.36 \\
\hline W590 & 83.94 & 79.00 & 8.00 \\
\hline W840 & 79.9 & 73.69 & \\
\hline
\end{tabular}


2020, Instituto Mexicano de Tecnología del Agua

Tecnología y

Open Access bajola licencia CC BY-NC-SA 4.0

(https://creativecommons.org/licenses/by-nc-sa/4.0/)

\begin{tabular}{|c|c|c|c|} 
La Higuera & 91.27 & 80.09 & 14.41 \\
\hline Río Chiquito & 82.7 & 72.57 & 13.06 \\
\hline Arroyo Blanco & 83.23 & 73.03 & 13.14 \\
\hline Arroyo Obispo & 90.19 & 79.14 & 14.24 \\
\hline
\end{tabular}

Para conocer el nivel de ajuste entre el hidrograma observado y calibrado se aplicaron pruebas de bondad de ajuste (Tabla 5). Estos parámetros, principalmente Nash-Sutcliffe (Moriasi et al., 2007), indican que el ajuste del modelo hidrológico está en un rango aceptable, por lo que se adoptaron los valores calibrados de valores de número de curva de escurrimiento para una posterior modelación hidrológica de eventos con duración de 24 horas, y para los periodos de retorno de 100 y 500 años.

Tabla 5. Pruebas de bondad de ajuste.

\begin{tabular}{|l|c|c|}
\hline \multicolumn{1}{|c|}{ Prueba de bondad } & Valor & Calificación \\
\hline Nash-Sutcliffe & 0.273 & Aceptable \\
\hline RMSE & 13.971 & --- \\
\hline Coeficiente de determinación $\left(r^{2}\right)$ & 0.743 & Aceptable \\
\hline
\end{tabular}

En la Figura 4 se presenta la comparación gráfica de los hidrogramas observado, simulado y calibrado. 


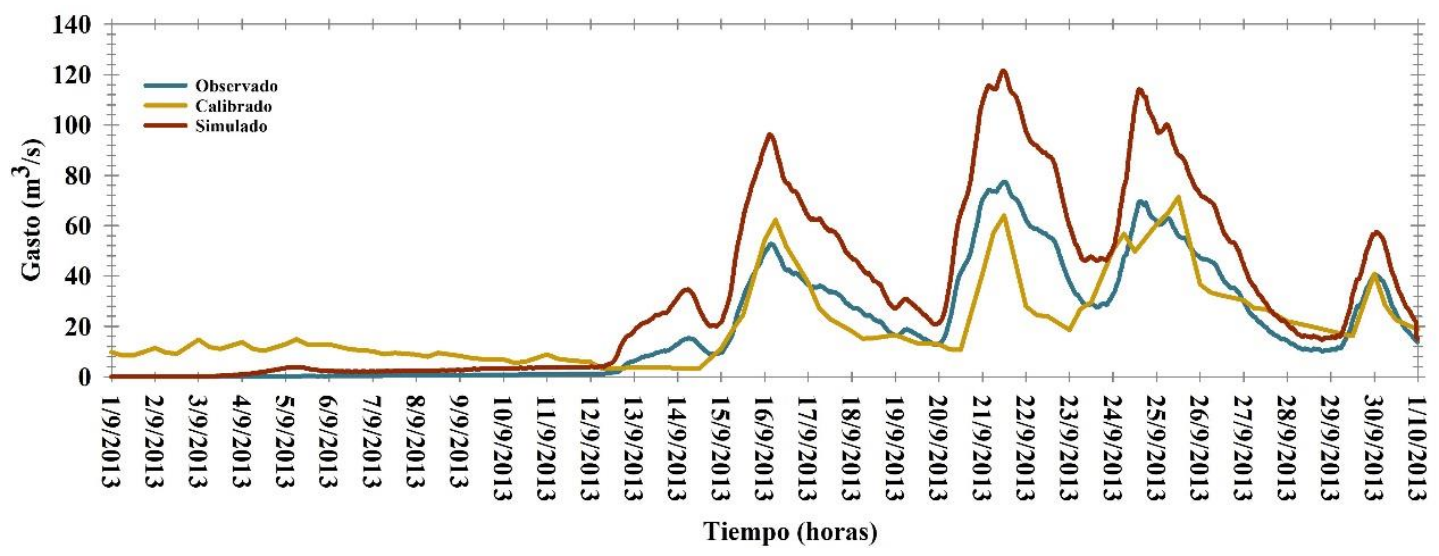

Figura 4. Comparación de hidrogramas observado, simulado y calibrado.

Con el modelo hidrológico calibrado se identificó un evento con duración de 24 horas, del 20 de septiembre a las 12:00 horas al 21 de septiembre a las 12:00 horas. Retomando los hietogramas adimensionales correspondientes al evento señalado (Figura 5), se multiplicaron por la lámina de precipitación asociada con los periodos de retorno de 100 y 500 años. 


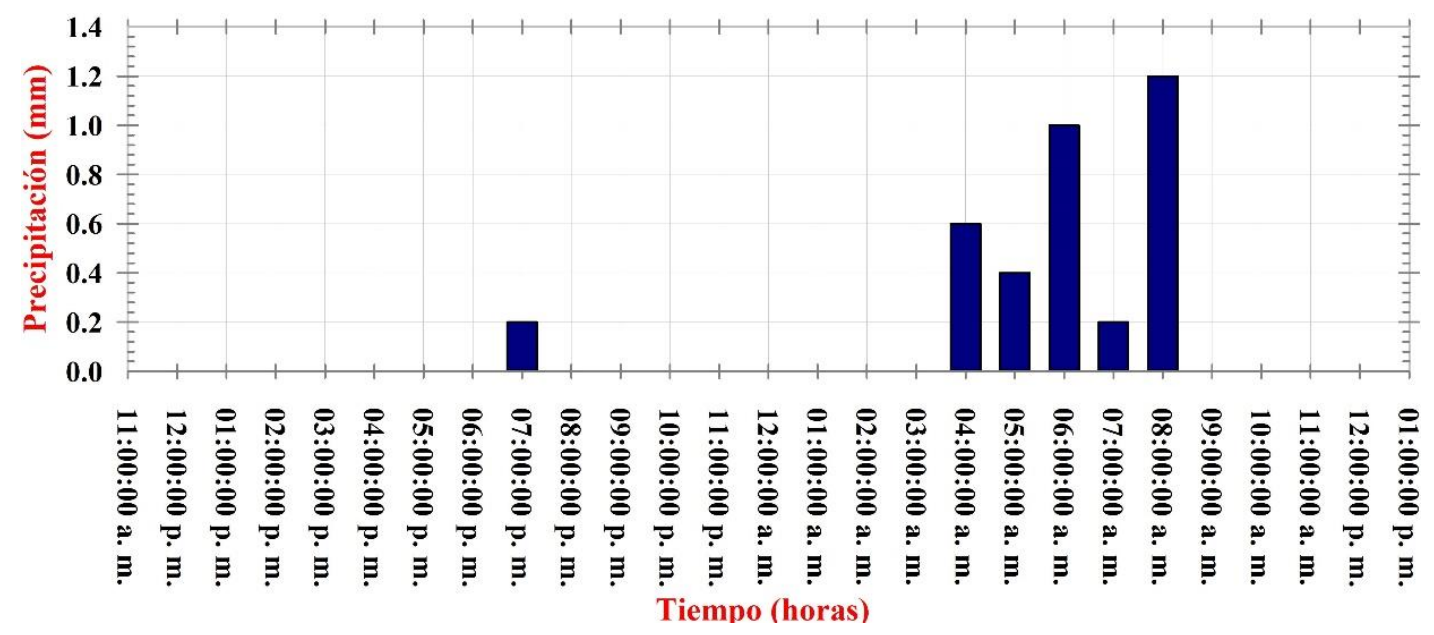

Figura 5. Hietograma con duración de 24 horas.

Se corrió el modelo hidrológico para los eventos con duración de 24 horas y obtuvieron los hidrogramas para cada subcuenca que, a su vez, corresponden a los cauces tributarios que aportan escurrimientos al río Grande en su tramo por la ciudad (Figura 6 y Figura 7).

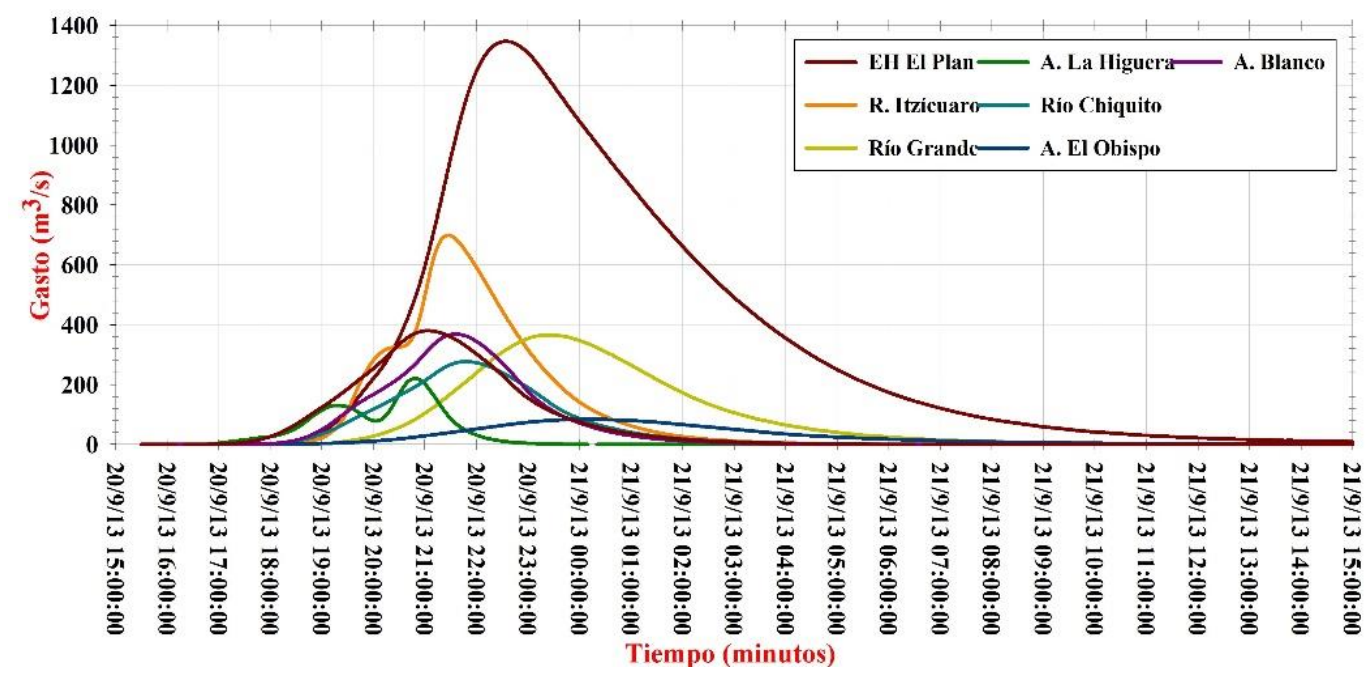

Figura 6. Hidrogramas para el periodo de retorno de 100 años. 


\section{0, Instituto Mexicano de Tecnología del Agua}

Tecnología y

Open Access bajo la licenciaCC BY-NC-SA 4.0

(https://creativecommons.org/licenses/by-nc-sa/4.0/)

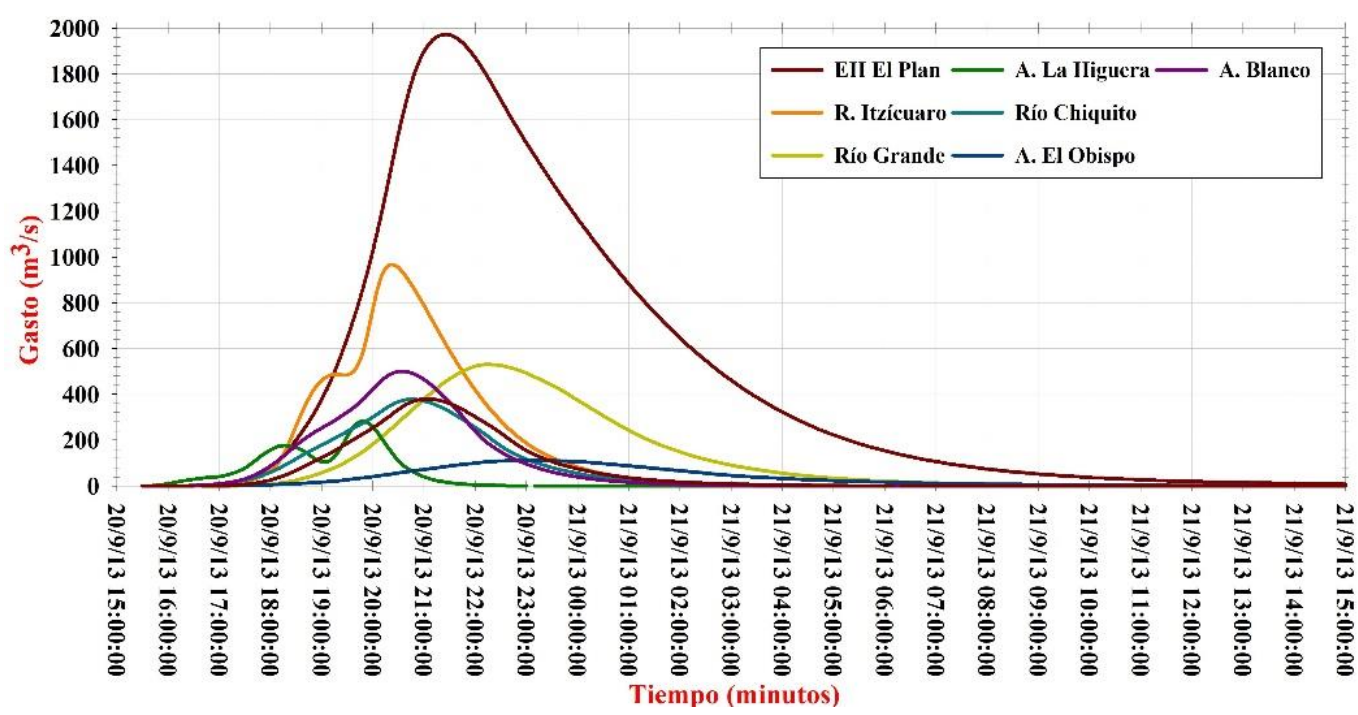

Figura 7. Hidrogramas para el periodo de retorno de 500 años.

\section{Modelación hidráulica}

La modelación hidráulica se realizó en el programa IBER (FLUMEN, 2017), utilizando la topografía levantada de modo directa en campo; para el cauce y llanuras de inundación se utilizó topografía LIDAR (Conagua, 2016).

El modelo hidráulico replicó la topografía mediante una malla de celdas triangulares; para la zona del cauce se asignó un tamaño de celda de cinco metros y para las llanuras de 10 metros. También se definieron

Tecnología y ciencias del agua, ISSN 2007-2422,11(3), 01-26. DOI: 10.24850/j-tyca-2020-0301 
los coeficientes de rugosidad de Manning: valores 0.18 para el cauce y en las Ilanuras de inundación de 0.032. El gasto de cada afluente se asignó con el correspondiente hidrograma para los periodos de retorno de $100 \mathrm{y}$ 500 años.

El modelo transitó las avenidas, y estimó los tirantes y cotas máximas alcanzadas por el agua en la zona del cauce y márgenes de los ríos. Con estos resultados, que pudieran esperase para los periodos de retorno modelados, las zonas vulnerables a inundaciones son evidentes (Figura 8 y Figura 9); tales zonas corresponden a colonias con gran población como Fraccionamiento San Lorenzo Itzícuaro, Ampliación del Club Campestre La Huerta, Molino de Parras, Profesor Jesús Romero Flores y la unidad deportiva Cuauhtémoc.

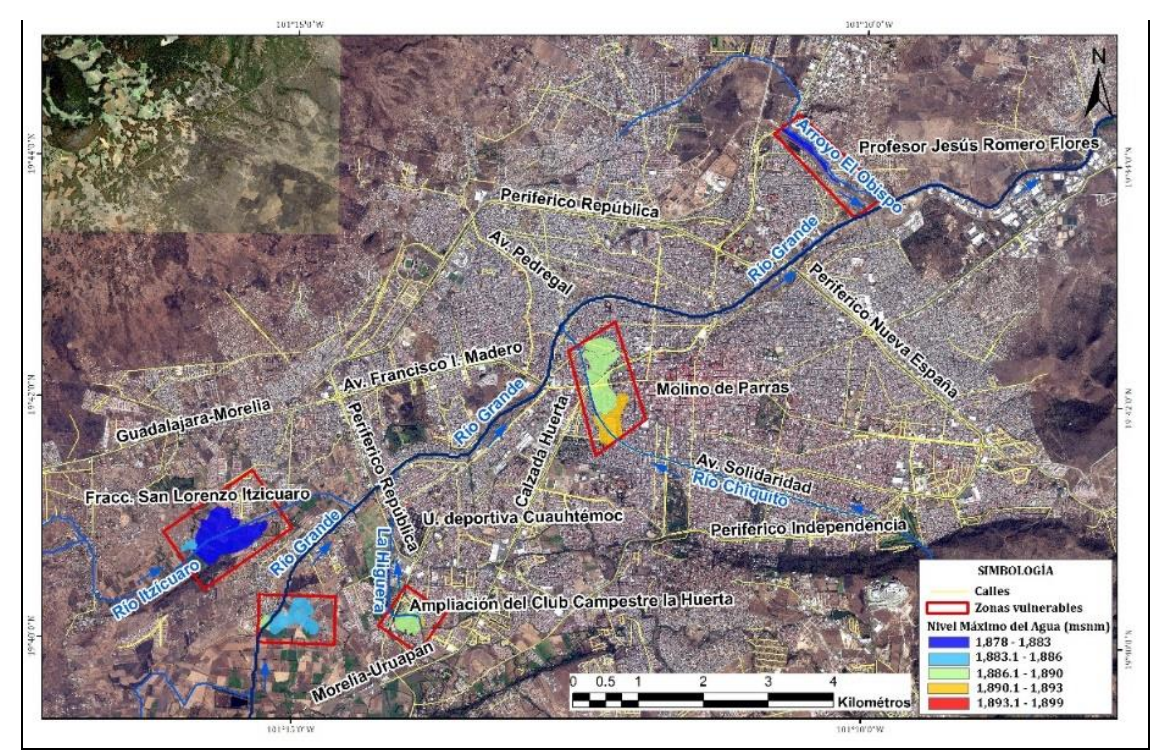

Figura 8. Visualización de la simulación hidráulica para $\operatorname{Tr}=100$ años. 


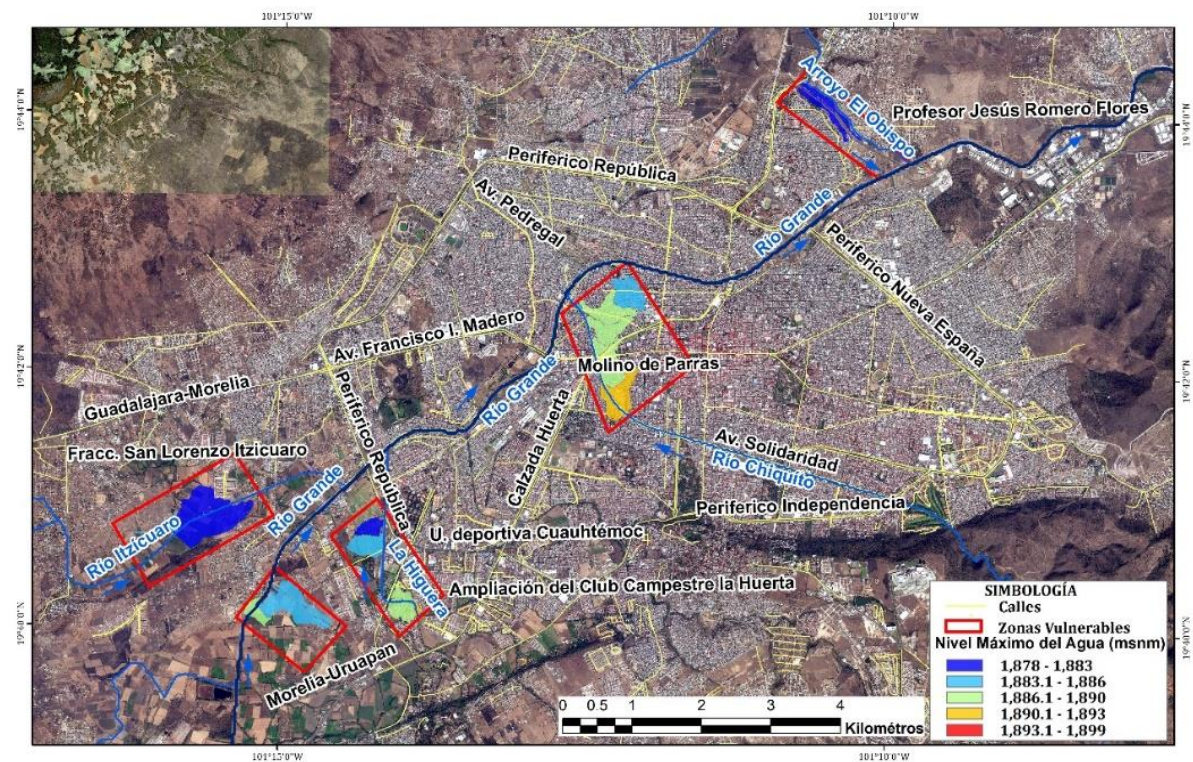

Figura 9. Visualización de la simulación hidráulica para $\operatorname{Tr}=500$ años.

\section{Discusión}

En lo referente al análisis de precipitaciones, se distribuyeron mediante hietogramas de las EMA aplicados a las EMC; en los últimos años se ha implementado el uso de radares (Méndez-Antonio, Magaña, Caetano, DaSilveira, \& Domínguez, 2009; Méndez-Antonio, Domínguez-Mora, Magaña-Rueda, \& Carrizosa-Elizondo, 2006; Magaña-Hernández, Bâ, \& Guerra-Cobián, 2013) y satélites (Zubieta, Getirana, Espinoza, Lavado, \& Aragón, 2017; Zubieta, Laqui, \& Lavado, 2018). Se decidió no utilizar Tecnología y ciencias del agua, ISSN 2007-2422, 11(3), 01-26. DOI: $10.24850 / j$-tyca-2020-0301 
Tecnología y

Ciencias $\stackrel{\unlhd}{\unlhd}$ Agua
2020, Instituto Mexicano de Tecnología del Agua

Open Access bajo la licencia CC BY-NC-SA 4.0

(https://creativecommons.org/licenses/by-nc-sa/4.0/)

estas dos tecnologías e implementar la señalada por López et al. (2012); sin embargo, es seguro que esas tecnologías se utilizarán en el futuro.

Las zonas vulnerables determinadas en esta investigación corresponden a los periodos de retorno de 100 y 500 años. CoronaMorales (2009) reporta lo publicado en el Atlas de Riesgos de Morelia (Figura 10), en el que se utiliza un modelo de distribución de la lámina de agua a cada $25 \mathrm{~cm}$; ello se llevó a cabo mediante un análisis de cotas y la identificación de zonas que resultaron inundadas en 2003; cabe señalar que en ese trabajo no se usó un modelo Iluvia-escurrimiento; pero al tratarse de una tormenta observada y la identificación de zonas afectadas, se logró considerar problemas derivados por el estancamiento del agua a consecuencia de la obstrucción por basura y desechos; evidentemente, las áreas en su mayoría no coinciden con lo determinado en este trabajo.

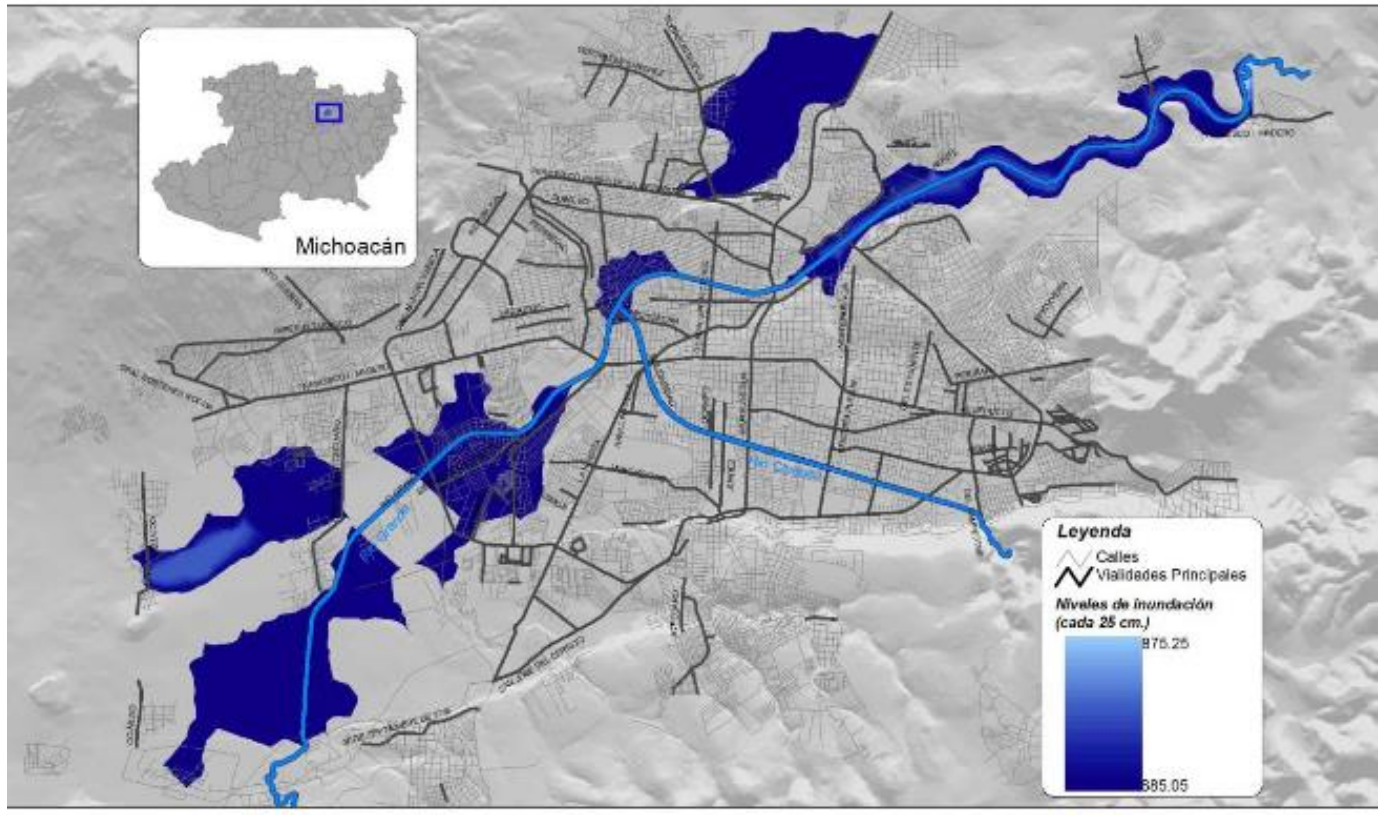




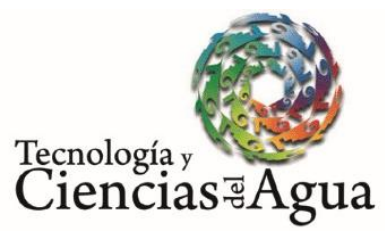

2020, Instituto Mexicano de Tecnología del Agua

Open Access bajola licencia CC BY-NC-SA 4.0

(https://creativecommons.org/licenses/by-nc-sa/4.0/)

Figura 10. Modelo de distribución de la lámina de agua a cada $25 \mathrm{~cm}$. Atlas de Riesgos de Morelia. Fuente: Corona-Morales (2009).

Otro trabajo fue llevado a cabo por Roblero-Hidalgo (2018), donde define áreas vulnerables a inundaciones mediante un modelo Iluviaescurrimiento y para un evento continuo desde enero de 2013 hasta enero de 2014. Dado que en el presente trabajo se utilizaron periodos de retorno, las áreas inundables coinciden, pero las estimadas para los periodos de retorno abarcan áreas más extensas.

\section{Conclusiones}

La metodología propuesta para identificar zonas vulnerables a inundaciones contempló el uso de modelos. El primero, hidrológico, en HEC-HMS, alimentado con hietogramas generados a partir de información registradas en las EMA y EMC, esquema de subcuencas, valores de curva número de escurrimientos y tiempos de retraso. Se calibró con base en un hidrograma aforado en la EHC El Plan y pruebas de bondad de ajuste. De acuerdo con Nash-Sutcliffe, resultó un modelo aceptable.

El otro modelo fue uno hidráulico, en IBER, al que se le suministraron los hidrogramas y una malla con la topografía; con este Tecnología y ciencias del agua, ISSN 2007-2422, 11(3), 01-26. DOI: 10.24850/j-tyca-2020-0301 
modelo se obtuvieron las zonas vulnerables a inundaciones en la ciudad de Morelia, Michoacán, para los periodos de retorno de 100 y 500 años.

Los modelos utilizados, HEC-HMS e IBER, son muy potentes y robustos para la estimación del proceso lluvia-escurrimiento y tránsito de avenidas en los cauces urbanos; sin embargo, requieren de información específica y de difícil acceso en México.

\section{Referencias}

Aparicio, M. F. (1992). Fundamentos de hidrología de superficie. México, DF, México: Limusa.

Conagua, Comisión Nacional del Agua. (2016). Actualización del estudio para el control de avenidas en el sistema Río Grande-río Chiquito de la presa Cointzio a su desembocadura al lago de Cuitzeo. Morelia, México: Dirección Local Michoacán.

Conagua, Comisión Nacional del Agua. (2011). Manual para el control de inundaciones. Recuperado de http://cenca.imta.mx/pdf/manualpara-el-control-de-inundaciones.pdf

Corona-Morales, N. (2009). Vulnerabilidad de la ciudad de Morelia a inundaciones (tesis de maestría). Centro de Investigaciones en Geografía Ambiental, Universidad Nacional Autónoma de México, México.

FLUMEN. (2017). IBER (2.4.3). Software para la modelación hidráulica en 2D. Recuperado de http://www.iberaula.es/space/54/downloads 
Tecnología y

Ciencias $\stackrel{\Xi}{\unlhd}$ gua
2020, Instituto Mexicano de Tecnología del Agua

Open Access bajo la licencia CC BY-NC-SA 4.0

(https://creativecommons.org/licenses/by-nc-sa/4.0/)

FLUMEN. (2010). Modelización bidimensional del flujo en lámina libre en aguas poco profundas. Manual de básico usuario 07.06.2010. Catalunya, España: Universidad Politécnica de Catalunya.

Fuentes-Mariles, O. A., \& Franco, V. (1999). Capítulo 3. Estudio hidrológico para obras de protección. En: Manual de ingeniería de ríos. México, DF, México: Instituto de la Ingeniería, Universidad Nacional Autónoma de México.

HEC-GeoHMS. (2017). Software para la preparación de datos de GIS para la importación a HEC-HMS (10.2). Recuperado de https://www.hec.usace.army.mil/software/hecgeohms/downloads.aspx

HEC-HMS. (2017). Software para la modelación hidrológica (4.2.1). Recuperado de http://www.hec.usace.army.mil/software/hechms/downloads.aspx

Kidd, C., \& Huffman G. (2011). Global precipitation measurement. Meteorological Applications. Royal Meteorological Society, 18, 334353. Recuperado de https://rmets.onlinelibrary.wiley.com/doi/pdf/10.1002/met.284

López, J., González, M., Scaini, A., Goñi, M., Valdenebro, J., \& Gimena, F. (2012). Caracterización del modelo HEC-HMS en la cuenca de río Arga en Pamplona y su aplicación a cinco avenidas significativas. Obras y Proyectos, 12, 15-30. Recuperado de https://scielo.conicyt.cl/pdf/oyp/n12/art02.pdf

Magaña-Hernández, F., Bâ, K., \& Guerra-Cobián, V. (2013). Estimación del hidrograma de crecientes con modelación determinística y 
precipitación derivada de radar. Agrociencia, 47, 739-752. Recuperado de http://www.scielo.org.mx/pdf/agro/v47n8/v47n8a1.pdf

Méndez-Antonio, B., Magaña, V., Caetano, E., Da-Silveira, R. B., \& Domínguez, R. (2009). Analysis of daily precipitation based on weather radar information in México City. Atmósfera, 22(2), 299313. Recuperado de http://www.scielo.org.mx/pdf/atm/v22n3/v22n3a5.pdf

Méndez-Antonio, B., Domínguez-Mora, R., Magaña-Rueda, R., \& Carrizosa-Elizondo, E. (2006). Calibración hidrológica de radares meteorológicos. Ingeniería hidráulica en México, 21(4), 43-64.

Moriasi, D., Arnold, J., Van-Liew, M. W., Bingner, R., Harmel, R., \& Veith, T. (2007). Model evaluation guidelines for systematic quantification of accuracy in watershed simulation. Transactions of the ASABE, 50 (3), 885-900.

Recuperado de https://www.researchgate.net/publication/43261199_Model_Evalua tion_Guidelines_for_Systematic_Quantification_of_Accuracy_in_Wat ershed_Simulations

Roblero-Hidalgo, R. (2018). Sistema de alerta temprana, para reducir daños de eventos hidrometeorológicos extremos (tesis de doctorado). Colegio de Postgraduados, Postgrado en Hidrociencias, Campus Montecillo, Texcoco, México.

USACE, U.S. Army Corps of Engineers. (2016). User's Manual of Hydrologic Modeling System HEC-HMS. Recuperado de 
2020, Instituto Mexicano de Tecnología del Agua

Tecnología y

Ciencias $₫$ Agua

http://www.hec.usace.army.mil/software/hec-

ms/documentation.aspx

WMO, World Meteorological Organization. (2011). Manual on flood forecasting and warning. Recuperado de http://www.library.wmo.int

Zubieta, R., Getirana, A., Espinoza, J. C., Lavado, W., \& Aragón, L., (2017). Hydrological modeling of the Peruvian-Ecuadorian Amazon Basin using GPM-IMERG satellite-based precipitation dataset. Hydrology and Earth System Sciences, 21(7), 3543-3555.

Zubieta, R., Laqui, W., \& Lavado, E. (2018). Modelación hidrológica de la cuenca del río Ilave a partir de datos de precipitación observada y de satélite, periodo 2011-2015, Puno, Perú. Tecnología y ciencias del agua, 9(5), 85-105, DOI:10.24850/j-tyca-2018-05-04. 